\title{
Control of 2D flexible structures by confinement of vibrations and regulation of their energy flow
}

\author{
Fakhreddine Landolsi ${ }^{\mathrm{a}}$, Slim Choura ${ }^{\mathrm{b}, *}$ and Ali H. Nayfeh ${ }^{\mathrm{c}}$ \\ ${ }^{a}$ Robotics and Intelligent Systems Laboratory, Department of Mechanical Engineering and Materials Science, Rice \\ University, Houston, TX 77005-1892, USA \\ ${ }^{\mathrm{b}}$ Applied Mechanics and Systems Research Laboratory, Tunisia Polytechnic School, B.P. 743, La Marsa 2078, \\ Tunisia \\ ${ }^{\mathrm{c}}$ Department of Engineering Science and Mechanics, MC 0219, Virginia Tech, Blacksburg, VA 24061, USA
}

\section{Received 21 June 2007}

Revised 7 December 2007

\begin{abstract}
In this paper, we investigate the control of 2D flexible structures by vibration confinement and the regulation of their energy flow along prespecified spatial paths. A discretized-model-based feedback strategy, aiming at confining and suppressing simultaneously the vibration, is proposed. It is assumed that the structure consists of parts that are sensitive to vibrations. The control design introduces a new pseudo-modal matrix derived from the computed eigenvectors of the discretized model. Simulations are presented to show the efficacy of the proposed control law. A parametric study is carried out to examine the effects of the different control parameters on the simultaneous confinement and suppression of vibrations. In addition, we conducted a set of simulations to investigate the flow control of vibrational energy during the confinement-suppression process. We found that the energy flow can be regulated via a set of control parameters for different confinement configurations.
\end{abstract}

Keywords: Confinement of vibration, 2D flexible structures, pseudo-modal matrix, flow control of vibrational energy

\section{Introduction}

Flexible structures can undergo vibratory motion that results from an initial energy distribution, persistent excitation, or both. The vibrations can reach unwanted structural resonances, and thus, damage the sensitive parts of the structure. Methods of vibration control may be grouped into three main categories: reduction at the source, isolation of the sensitive equipment or reduction of the response[1]. The reduction of the response can be achieved by altering the natural frequencies of the system to avoid potential resonances and introducing energy dissipation mechanisms.

The idea of vibration confinement originated from the concept of mode localization, which occurs mainly in slightly-perturbed periodic structures. Hodges [2] discussed the possibility of using structural irregularities for vibration confinement. Vakakis [3] investigated passive spatial confinement of impulsive responses. Allaei [4] compared passive confinement similar to the mode localization phenomenon and conventional control techniques. The use of passive methods presents two limitations: they are effective only for high frequencies and cannot be applied to strongly-modified structures.

Active confinement methods are based on feedback techniques to achieve eigenstructure assignment for the purpose of vibration suppression. Many algorithms were proposed to assign arbitrary eigenvalues and eigenvectors to flexible

\footnotetext{
*Corresponding author. E-mail: s_choura@yahoo.com.
} 
structures via feedback. Fahmy and O'Reilly [5] examined the eigensystem alteration of linear multivariable systems. Kautsy et al. [6] proposed a robust pole assignment technique using linear state feedback. Robust eigenstructure assignment for flexible structures was investigated by Juang et al. [7]. Maghami et al. [8,9] included dissipativity constraints for the eigensystem assignment using output feedback. Shelley and Clark [10] used singular value decomposition to develop an eigenvector scaling algorithm. Only few eigenstructure assignment algorithms were applied to the problem of vibration confinement.

Song and Jayasuriya [11] developed an algorithm for eigenvector assignment where the specification of special mode shapes was shown to alter the distribution of the vibrational energy. Shaw and Jayasuriya [12] and Clark and Shelley [13] analyzed active mode localization in distributed-parameter systems by altering their eigenstructure. The robustness and margin stability of such techniques depend highly on the dynamic model and actuator-sensor placement considerations. Preumont [14] showed that the use of collocated actuators and sensors guarantees the asymptotic stability of a wide class of single-input single-output control systems. In fact, for lightly-damped structures, collocated actuator and sensor pairs lead to alternating poles and zeros near the imaginary axis on the left half $s$-plane even if the system parameters are subject to large perturbations.

Meyer and Collet [15] proposed a control design to achieve vibration isolation. The relative displacement and transmitted force between the sensitive element and its disturbing support are employed as feedback signals. The simplicity of the proposed control law enables optimization of the different design parameters. Other studies investigated means to maintain the vibrational energy near the excitations points and to prevent it from propagating to the sensitive parts [16]. Choura and Yigit [17] developed a control strategy for the confinement of vibrations in flexible structures. This strategy is based on applying distributed feedback to continuous structures. Feedback forces are used to alter the original mode shapes and to convert them to exponentially-decaying functions of the space variable. As a consequence, parts of the structure reach their equilibrium at faster rates at the expense of slowing down convergence of the remaining parts to zero. Choura and Yigit [18] proposed also a similar method based on FEM reduced-order systems.

To the best of our knowledge, no research studies have investigated the confinement of vibrations and the flow control of vibrational energy in flexible structures. In fact, the majority of studies on vibration confinement addressed its efficiency on elementary structures, such as bars and beams. In this paper, we propose a design strategy for the confinement and flow control of vibrations in 2D flexible structures. Special interest is given to controlling the flow of vibrational energy and isolating the sensitive parts of the structure. The controller design is based on discretepoint actuators and uses a pseudo-modal matrix for vibration confinement. The reduced-order system is obtained using the Harmonic Quadrature Method (HQM) [19-22]. This latter was shown to yield accurate results with less computational cost as opposed to other classical discretization techniques. We also propose that the pseudo-modal matrix be determined using the eigenvectors associated with the HQM-discretized model. Simulations are carried out in order to investigate the effects of different design parameters on the confinement of vibrations. The use of the resulting pseudo-modal matrix is contrasted to classical pseudo-modal matrices. Another set of simulations is conducted to demonstrate the possibility of controlling the flow of vibrational energy.

\section{Model discretization of 2D flexible structures}

The proposed active confinement strategy is based on the use of point force actuators. As a result, a discretization technique is needed for order reduction of the system dynamics.

\subsection{Modeling flexural vibrations of $2 D$ structures}

In this study, we consider the class of 2D flexible structures described by the following linear partial differential equation and set of boundary conditions:

$$
\begin{aligned}
& \mathcal{M}\left[\frac{\partial^{2} w}{\partial t^{2}}(P, t)\right]+\mathcal{C}\left[\frac{\partial w}{\partial t}(P, t)\right]+\mathcal{K}[w(P, t)]=f(P, t)+d(P, t) \\
& \mathcal{B}_{i}[w(P, t)]=0 \quad i=1,2, \cdots, p
\end{aligned}
$$


where $\mathcal{M}, \mathcal{C}$ and $\mathcal{K}$ are the linear mass, damping and stiffness differential operators. The independent variable $t$ is time, $P$ is a point of the spatial domain $D$, and for every point on the boundary $S, \mathcal{B}_{i}$ is a set of linear differential operators characterizing the boundary conditions, and $f$ is a distributed feedback force. It is assumed that the structure vibrates under the influence of an external disturbance $d(P, t)$ and that the distributed load $f(P, t)$ is the essential mechanism for vibration confinement and suppression.

In this study, we adopt HQM for discretizing the class of 2D structures described by Eq. (1). In the literature, HQM has been proved to yield better results with less computational cost than the Finite Element Method [20]. The basic idea of HQM is that the derivative of a function, with respect to the space variable at a given sampling point, is approximated as a weighted linear sum of the values of the function at the sampling points in the domain of that variable. In case of $2 \mathrm{D}$ problems, this can be characterized by the following relation:

$$
\left.\frac{\partial^{(r+s)} g}{\partial \xi^{r} \partial \eta^{s}}\right|_{\left(\xi_{\left.i, \eta_{j}\right)}\right.}=A^{(\xi, r)} G\left[A^{(\eta, s)}\right]^{T}
$$

where $G=\left[g\left(\xi_{i}, \eta_{j}\right)\right]_{\left(N_{\xi} \times N_{\eta}\right)}, A^{(\xi, r)}=\left[A_{i j}^{(\xi, r)}\right]_{\left(N_{\xi} \times N_{\xi}\right)}$ and $A^{(\eta, s)}=\left[A_{i j}^{(\eta, s)}\right]_{\left(N_{\eta} \times N_{\eta}\right)} . N_{\xi}$ and $N_{\eta}$ represent the numbers of grid points along the $\xi$ and $\eta$ directions, respectively. The expression of the HQM weighting coefficients can be found in [19] $\left(A^{(\xi, 0)}\right.$ and $A^{(\eta, 0)}$ are equal to the identity matrix).

The resulting HQM-discretized system can be written in the following form:

$$
M \ddot{X}+C \dot{X}+K X=B^{c t r} f+B^{d i s} d
$$

where $M, C$ and $K$ are respectively the inertia, damping and stiffness matrices, $B^{c r t}$ and $B^{d i s}$ are the input distribution matrices associated with the control and disturbance signals, and $X=\left[X_{i, j}\right]$ is a matrix representing the structure deflection at the grid points. In order to recast the system into the conventional form where $X$ is a vector, we use the fact that for $A \in M_{m, n}, B \in M_{p, q}$ and $X \in M_{n, p}$ we have [23]:

$$
\operatorname{vec}(A X B)=\left(B^{T} \otimes A\right) \operatorname{vec}(X)
$$

where $\operatorname{vec}(X)$ is the vector-function of the rectangular matrix $X$ formed by stacking its columns into one vector, and $\otimes$ denotes the Kronecker product.

\subsection{Bending of plates}

Without loss of generality, this paper considers a plate undergoing flexural vibration, as an example of 2D structures, to illustrate the proposed control design for confining its vibratory motion and regulating its energy flow. According to the classical plate theory, the flexural vibrations of a rectangular plate can be described by the following nondimensional partial differential equation:

$$
\frac{h^{3}}{6 L_{x}^{3}}\left(\frac{\partial^{4} v^{*}}{\partial \xi^{4}}+2 r^{2} \frac{\partial^{4} v^{*}}{\partial \xi^{2} \partial \eta^{2}}+r^{4} \frac{\partial^{4} v^{*}}{\partial \eta^{4}}\right)+\frac{\partial^{2} v^{*}}{\partial \tau^{2}}=\frac{h^{2} L_{x}}{6 D}\left(\sum_{k=1}^{n_{u}} f_{k}(\tau) \delta\left(\xi-\xi_{k}\right) \delta\left(\eta-\eta_{k}\right)\right)
$$

where $\xi=\frac{x}{L_{x}}, \eta=\frac{y}{L_{y}}, v^{*}=\frac{v}{h}, \tau=\sqrt{\frac{6 D}{\rho L_{x} h^{4}}} t, r=\frac{L_{x}}{L_{y}}$ and $D=\frac{E h^{3}}{12\left(1-\nu^{2}\right)} . L_{x}$ and $L_{y}$ are the plate sides, $h$ is the thickness, $\rho$ is the material density, $E$ is the modulus of elasticity, $\nu$ is the Poisson's ratio, and $f_{k}\left(k=1,2, \ldots, n_{u}\right)$ are the amplitudes of the $n_{u}$ discrete actuators. Applying the HQM to Eq. (5) yields the following discretized plate dynamics:

$$
\begin{aligned}
& \frac{h^{3}}{6 L_{x}^{3}}\left[\left(I_{N_{\eta}} \otimes A^{(\xi, 4)}\right)+2 r^{2}\left(A^{(\eta, 2)} \otimes A^{(\xi, 2)}\right)+r^{4}\left(A(\eta, 4) \otimes I_{N_{\xi}}\right)\right] \operatorname{vec}(V) \\
& \quad+\left(I_{N_{\eta}} \otimes I_{N_{\xi}}\right) \operatorname{vec}(\ddot{V})=\frac{h^{2} L_{x}}{6 D}\left(I_{N_{\eta}} \otimes B\right) \operatorname{vec}(F)
\end{aligned}
$$

where $V=\left[V_{i j}=v^{*}\left(\xi_{i}, \eta_{j}\right)\right], \ddot{V}=\left[\ddot{V}_{i j}=\frac{\partial^{2} v^{*}\left(\xi_{i}, \eta_{j}\right)}{\partial \tau^{2}}\right]$ and $F=\left[F_{k}=f_{k}(\tau)\right] . I_{N_{\xi}}$ and $I_{N_{\eta}}$ denote the identity matrices of size $N_{\xi} \times N_{\xi}$ and $N_{\eta} \times N_{\eta}$, respectively. Equation (6) can be rewritten as: 


$$
M \ddot{X}+K X=B^{c t r} f
$$

where $X=\operatorname{vec}(V), \ddot{X}=\operatorname{vec}(\ddot{V})$ and $f=\operatorname{vec}(F)$.

Next, we present a control strategy for $2 \mathrm{D}$ structures by the confinement of vibrations. The proposed design uses HQM to determine the actuator locations and compute a pseudo-modal matrix, a key parameter for vibration confinement.

\section{Proposed active controller for the confinement of vibrations}

The proposed control strategy addresses two important design issues:

1. Is it possible to isolate sensitive parts of a 2D structure subject to external and/or internal excitations?

2. Can the flow path(s) of the confined vibratory motion within the structure be controlled? If yes, then what would be the most convenient flow path(s) for accelerating the vibration confinement with less input amplitudes?

In order to answer the above concerns, let the discretized system, described by Eq. (8), be decomposed into two subsystems: the first one includes all sensitive parts in addition to at least one neighboring nonsensitive part and the second includes the remaining parts. A sensitive part is characterized by amplitude of vibration that must be kept low as compared to that of a nonsensitive part. The decomposed system is described by:

$$
\left[\begin{array}{ll}
M_{p p} & M_{p r} \\
M_{r p} & M_{r r}
\end{array}\right]\left[\begin{array}{l}
\ddot{X}_{p} \\
\ddot{X}_{r}
\end{array}\right]+\left[\begin{array}{ll}
K_{p p} & K_{p r} \\
K_{r p} & K_{r r}
\end{array}\right]\left[\begin{array}{c}
X_{p} \\
X_{r}
\end{array}\right]=\left[\begin{array}{cc}
I_{p p} & 0_{p k} \\
0_{(n-p) p} & B_{(n-p) k}
\end{array}\right]\left[\begin{array}{l}
f_{p} \\
f_{k}
\end{array}\right]
$$

where $p$ and $r$ are the orders of the first and second subsystems, respectively, with $p+r=n$ and $p+k=n_{u} . f_{p}$ and $f_{k}$ are the sets of control inputs associated with the first and second subsystems, respectively. The key role of this decomposition is to decouple the first subsystem from the second one. The main advantage of this control over classical feedback control is the possibility of imposing separate convergence rates on the sensitive and nonsensitive parts. In fact, this becomes possible through the use of the feedback inputs $f_{p}$ and $f_{k}$. The main task of $f_{p}$ is to redistribute and suppress the vibrational energy in the first subsystem. The second feedback input $f_{k}$ acts mainly as a stabilizer for the second subsystem. Reducing the number of actuators is equivalent to minimizing the size of $f_{k}$ provided that the second subsystem remains stable. Next, the design of each of these input vectors is described.

\subsection{Design of $f_{p}$}

The synthesis of $f_{p}$ requires the use of $p$ actuators, where $p$ corresponds to the dimension of the first subsystem. The control $f_{p}$ allows the designer to put more emphasis on the sensitive parts by accelerating their convergence rates. In order to decouple the $X_{p}$ dynamics from that of $X_{r}, f_{p}$ is proposed to be given by:

$$
f_{p}(t)=M_{p r} \ddot{X}_{r}+K_{p r} X_{r}-\bar{M}_{p p} \ddot{X}_{p}-\bar{C}_{p p} \dot{X}_{p}-\bar{K}_{p p} X_{p}
$$

Substituting Eq. (9) into Eq. (8) yields:

$$
\left(M_{p p}+\bar{M}_{p p}\right) \ddot{X}_{p}+\bar{C}_{p p} \dot{X}_{p}+\left(K_{p p}+\bar{K}_{p p}\right) X_{p}=0
$$

Feedback is primarily used to alter the eigenstructure of the first subsystem with the aim of suppressing and confining the structural vibrations. Without loss of generality, such vibrations are assumed to result from external disturbances. The emphasis here is on designing stabilizing feedback matrices $\bar{M}_{p p}, \bar{C}_{p p}$ and $\bar{K}_{p p}$ that confine and eliminate the vibratory motion of the structure.

Let $X_{p}=Q \lambda_{p}$, where $Q$ is the pseudo-modal matrix and $\lambda_{p}$ is the vector of modal amplitudes associated with the first subsystem. Thus, Eq. (10) becomes:

$$
\ddot{\lambda}_{p}+\Gamma \dot{\lambda}_{p}+\Lambda \lambda_{p}=0
$$

where $\Gamma$ and $\Lambda$ are diagonal matrices defined as:

$$
\Gamma=\operatorname{diag}\left\{2 \zeta_{1} \omega_{1}, 2 \zeta_{2} \omega_{2}, \cdots, 2 \zeta_{n} \omega_{n}\right\} \quad=Q^{-1}\left(M_{p p}+\bar{M}_{p p}\right)^{-1} \bar{C}_{p p} Q
$$




$$
\Lambda=\operatorname{diag}\left\{\omega_{1}^{2}, \omega_{2}^{2}, \cdots, \omega_{n}^{2}\right\}=Q^{-1}\left(M_{p p}+\bar{M}_{p p}\right)^{-1}\left(K_{p p}+\bar{K}_{p p}\right) Q
$$

The coefficients $\zeta_{i}$ and $\omega_{i}(i=1,2, \ldots, p)$ are the damping ratios and natural frequencies associated with the controlled structure. The matrices $Q, \Gamma$ and $\Lambda$ are considered to be the design parameters for the synthesis of the control law. In particular, the matrix $Q$ is the main tool for the confinement of the vibrational energy in regions of the system domain. The choice of its rows decides the way the vibrational energy is reallocated in the system domain. The masses or nodes, which have to experience lower energy levels, must have the elements of their corresponding rows small. The form of $Q$ proposed in [24] is given by

$$
Q_{C}=\left[\begin{array}{ccccc}
1 & 1 & \cdots & 1 & 1 \\
\beta_{1} & \beta_{1} & \cdots & \beta_{1} & s_{2, p} \beta_{1} \\
\beta_{2} & \beta_{2} & \cdots & s_{3, p-1} \beta_{2} & s_{3, p} \beta_{2} \\
\vdots & \vdots & \vdots & \vdots & \vdots \\
\beta_{p-2} & \beta_{p-2} & \cdots & s_{p-1, p-1} \beta_{p-2} & s_{p-1, p} \beta_{p-2} \\
\beta_{p-1} & s_{p, 2} \beta_{p-1} & \cdots & s_{p, p-1} \beta_{p-1} & s_{p, p} \beta_{p-1}
\end{array}\right]
$$

where the coefficients $s_{i, j}$ are defined by:

$$
s_{i, j}= \begin{cases}(-1)^{p+1} & \text { if }(i+j) \text { is even } \\ (-1)^{p} & \text { if }(i+j) \text { is odd }\end{cases}
$$

These are introduced to maintain a pattern of sign changes associated with the columns of $Q_{C}$, and the scalars $\beta_{i}$ set the level of vibrational energy at each node.

In this study, we propose a new form of the pseudo-modal matrix $Q_{N}$ constructed with the aid of the HQMestimated modal matrix denoted by $W$. The proposed pseudo-modal matrix is given by:

$$
Q_{N}=\left[\begin{array}{ccccc}
\beta_{1,1} \frac{W_{1,1}}{\omega_{11}} & \beta_{1,2} \frac{W_{1,2}}{\omega_{12}} & \cdots & \beta_{1, p-1} \frac{W_{1, p-1}}{\omega_{1}(p-1)} & \beta_{1, p} \frac{W_{1, p}}{\omega_{1 p}} \\
\beta_{2,1} \frac{W_{2,1}}{\omega_{11}} & \beta_{2,2} \frac{W_{2,2}}{\omega_{12}} & \cdots & \beta_{2, p-1} \frac{W_{2, p-1}}{\omega_{1(p-1)}} & \beta_{2, p} \frac{W_{2_{1}, p}}{\omega_{p}} \\
\beta_{3,1} \frac{W_{3,1}}{\omega_{11}} & \beta_{3,2} \frac{W_{3,2}}{\omega_{12}} & \cdots & \beta_{3, p-1} \frac{W_{3, p-1}}{\omega_{1(p-1)}} & \beta_{3, p} \frac{W_{3, p}}{\omega_{1 p}} \\
\vdots & \vdots & \vdots & \vdots & \vdots \\
\beta_{p-1,1} \frac{W_{p-1,1}}{\omega_{11}} & \beta_{p-1,2} \frac{W_{p-1,2}}{\omega_{12}} & \cdots & \beta_{p-1, p-1} \frac{W_{p-1, p-1}}{\omega_{1(p-1)}} & \beta_{p-1, p} \frac{W_{p-1, p}}{\omega_{1 p}} \\
\beta_{p, 1} \frac{W_{p, 1}}{\omega_{11}} & \beta_{p, 2} \frac{W_{p, 2}}{\omega_{12}} & \cdots & \beta_{p, p-1} \frac{W_{p, p-1}}{\omega_{1(p-1)}} & \beta_{p, p} \frac{W_{p, p}}{\omega_{1 n}}
\end{array}\right]
$$

Although it can be used for 2D structures, the pseudo-modal matrix $Q=Q_{C}$ proposed in [24] was originally constructed for 1D structures. The proposed pseudo-modal matrix $Q_{N}$ relies on a more faithful representation of the eigensystem of the HQM-discretized structure. In addition, the $\beta_{i, j}$ coefficients, which define the way the vibrational energy is reallocated in the structure, can be separately specified at a given node associated with one of the mode shapes. This property allows more flexibility for both the vibration confinement and the control of energy flow. The advantages of the proposed form of the pseudo-modal matrix, as contrasted to $Q_{C}$, are addressed in the simulation study, provided later.

A closed-form expression for the displacement vector $X_{p}$ can be derived from Eq. (11). It is given by:

$$
X_{p}(t)=Q_{N}\left[H_{1}(t) Q_{N}^{-1} X_{p}(0)+H_{2}(t) Q_{N}^{-1} \dot{X}_{p}(0)\right]
$$

where $H_{1}(t)$ and $H_{2}(t)$ are time-varying diagonal matrices whose elements are given by:

$$
\begin{aligned}
& \left(H_{1}\right)_{i i}(t)=e^{-\xi_{i} \omega_{i} t}\left(\cos \left(\omega_{i} \sqrt{1-\xi_{i}^{2}} t\right)+\frac{\xi_{i}}{\sqrt{1-\xi_{i}^{2}}} \sin \left(\omega_{i} \sqrt{1-\xi_{i}^{2}} t\right)\right) \\
& \left(H_{2}\right)_{i i}(t)=e^{-\xi_{i} \omega_{i} t}\left(\frac{1}{\omega_{i} \sqrt{1-\xi_{i}^{2}}} \sin \left(\omega_{i} \sqrt{1-\xi_{i}^{2}} t\right)\right)
\end{aligned}
$$

Notice that the reallocation of the vibrational energy is ensured by $Q_{N}$ and the damping rate is regulated through the damping terms $\xi_{i} \omega_{i}(i=1,2, \cdots, p)$. 


\subsection{Design of $f_{k}$}

The main reason behind the use of the input $f_{k}$ is to ensure closed-loop stability of the second subsystem. As it is clear from the decomposition of the system dynamics, the feedback input $f_{k}$ does not influence the first subsystem. The stability of the second subsystem using $f_{k}$ is based on the following dynamics:

$$
M_{r r} \ddot{X}_{r}+K_{r r} X_{r}=B_{r k} f_{k}
$$

Using the state vector $x=\left[\begin{array}{c}X_{r} \\ \dot{X}_{r}\end{array}\right]$, Eq. (19) can be expressed in the following state space form:

$$
\dot{x}=\left[\begin{array}{cc}
0_{r r} & I_{r r} \\
-M_{r r}^{-1} K_{r r} & 0_{r r}
\end{array}\right] x+\left[\begin{array}{c}
0_{r k} \\
M_{r r}^{-1} B_{r k}
\end{array}\right] f_{k}
$$

It is required that the actuators be placed appropriately such that the second subsystem is controllable. The feedback input $f_{k}$ can be expressed in the form

$$
f_{k}=-\left[K_{1} K_{2}\right] x
$$

where the matrices $K_{1}$ and $K_{2}$ are determined by pole placement or linear quadratic regulation methods. Integrating the expressions of the feedback inputs $f_{p}$ and $f_{k}$ in the original system, we obtain:

$$
\left[\begin{array}{cc}
M_{p p} & 0_{p r} \\
M_{r p} & M_{r r}
\end{array}\right]\left[\begin{array}{c}
\ddot{X}_{p} \\
\ddot{X}_{r}
\end{array}\right]+\left[\begin{array}{cc}
C_{p p} & 0_{p r} \\
0_{r p} & B_{r k} K_{2}
\end{array}\right]\left[\begin{array}{c}
\dot{X}_{p} \\
\dot{X}_{r}
\end{array}\right]+\left[\begin{array}{cc}
K_{p p} & 0_{p r} \\
K_{r p} & K_{r r}+B_{r k} K_{1}
\end{array}\right]\left[\begin{array}{c}
X_{p} \\
X_{r}
\end{array}\right]=\left[\begin{array}{c}
0_{p} \\
0_{r}
\end{array}\right]
$$

Note that if the design parameters $Q_{N}, \Gamma$ and $\Lambda$ are specified, the relations between the feedback gain matrices $\bar{M}_{p p}$, $\bar{C}_{p p}$ and $\bar{K}_{p p}$ can be determined from Eq. (12) and Eq. (13). They are given by:

$$
\begin{aligned}
& \bar{C}_{p p}=\left(M_{p p}+\bar{M}_{p p}\right) Q_{N} \Gamma Q_{N}^{-1} \\
& \bar{K}_{p p}=-K_{p p}+\left(M_{p p}+\bar{M}_{p p}\right) Q_{N} \Lambda Q_{N}^{-1}
\end{aligned}
$$

Here, it should be emphasized that there are infinitely many sets of $\bar{M}_{p p}, \bar{C}_{p p}$ and $\bar{K}_{p p}$ matrices, all of which lead to the same structural response [25]. Therefore, the sign of these matrices can be controlled by proper choice of $Q_{N}, \Gamma$ and $\Lambda$. Inspection of Eqs (10) and (22) depicts that the control laws are constructed from knowledge of the displacement, velocity and acceleration vectors. Solving Eq. (10) for $\ddot{X}_{p}$ and then substituting the result into Eq. (9) yields a control law $f_{p}$ that can be made of a linear combination of the displacement and velocity vectors only. However, the implementation of the proposed control law needs the full observation of the displacement, velocity and acceleration vectors. In practice, the simultaneous measurements of the primal (displacement and velocity) and dual (acceleration) variables are impossible, as stated by the Heisenberg uncertainty principle. Thus, the implementation of such system requires the estimation of some of these variables using signal filters or estimation techniques.

In case of a full set of actuators, the implementation of the proposed confinement strategy assumes the use of collocated actuators and sensors. The choice of where to place these devices depends heavily on the assignment of the sensitive and nonsensitive parts of the structure. For a reduced number of actuators, the proposed strategy implies a full state measurement. The spatial region, which gathers the sensitive parts and one neighboring nonsensitive part, necessitates collocated actuator-sensors pairs. Whereas the region, which encloses the remainder of the nonsensitive parts, requires more sensors than actuators, and thus, the problem of spillover is likely to occur.

\section{Simulation study}

The main objective of the presented simulations is to demonstrate the ability of the proposed control strategy to confine the vibratory motion in 2D structures. The effects of the different design parameters, namely the matrices $\Lambda, \Gamma$ and $Q_{N}$ on the structural performance, are investigated. In addition, comparing the confinement performances using the pseudo-modal matrices $Q_{C}$ and $Q_{N}$ is worth investigating. Also, both cases of collocated (full set of actuators) and uncollocated (reduced set of actuators) actuator-sensor pairs are discussed. A simulation study is 


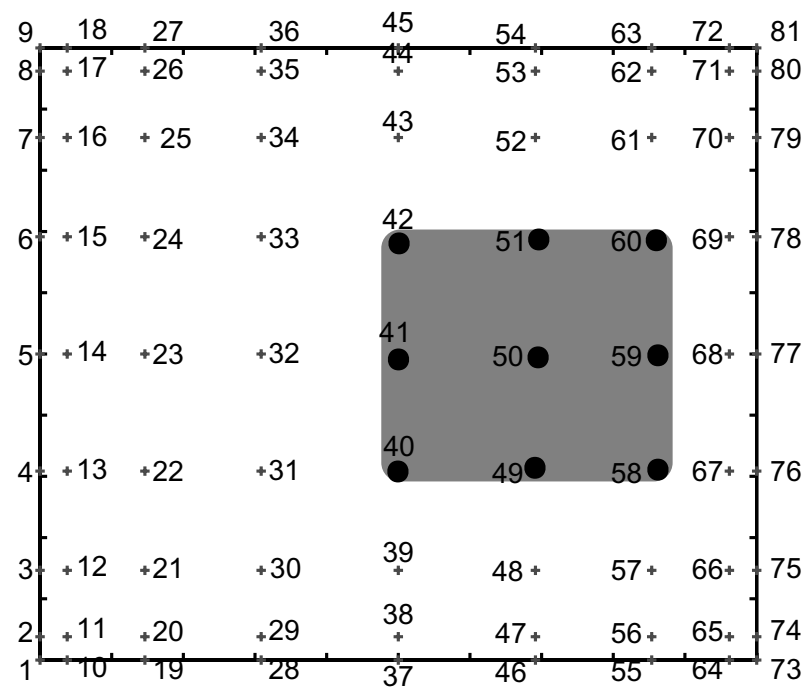

Fig. 1. Chebyshev-Gauss-Lobatto $9 \times 9$ grid.

carried out to examine the flow of the vibrational energy during the control process. All simulations are conducted on a square plate subjected to impact-type excitations applied to the sensitive parts. Chebyshev-Gauss-Lobatto sampling points are used for the $\xi$ and $\eta$ directions. These points are determined from

$$
\delta_{i}=\frac{1}{2}\left[1-\cos \left(\pi \frac{2 i-1}{N_{\delta}-1}\right)\right] \quad i=1,2, \ldots, N_{\delta} \quad ; \quad \delta=\xi, \eta
$$

\subsection{Full Set of Actuators (FSA)}

We consider a simply supported plate such that $L_{x}=L_{y}=1 \mathrm{~m}, h=5 \times 10^{-3} \mathrm{~m}, \rho=2730 \mathrm{~kg} \mathrm{~m}^{-3}$, $E=7 \times 10^{10} N m^{2}$ and $\nu=0.33$. A $9 \times 9$ grid is used to discretize the structure (see Figure 1). The sensitive region of the plate is represented in gray.

The present configuration implies the use of collocated actuators and sensors. The HQM solution of the corresponding eigenvalue problem agrees well with the exact solution given by $\omega_{i j}=\sqrt{\frac{h^{2} E}{12 \rho\left(1-v^{2}\right)}}\left(\left(\frac{i \pi}{L_{x}}\right)^{2}+\left(\frac{j \pi}{L_{y}}\right)^{2}\right)$. Let the plate be initially deflected such that:

$$
\left.\left[\begin{array}{lll}
X_{42} & X_{51} & X_{60} \\
X_{41} & X_{50} & X_{59} \\
X_{40} & X_{49} & X_{58}
\end{array}\right]\right|_{\tau=0}=\left[\begin{array}{ccc}
1 & 1 & 1 \\
1 & 1.5 & 1 \\
1 & 1 & 1
\end{array}\right]
$$

and zero everywhere else. In addition, the initial velocity at each grid point is taken zero. All damping coefficients are set to 0.3 and the natural frequencies of the original structure are unaltered after feedback.

The $\beta_{i j}$ coefficients associated with the sensitive nodes are set to 0.01 , whereas those associated with the nonsensitive region are set to 1 . Figure 2 displays the resulting plate time responses at selected nodes using the pseudo-modal matrices $Q_{C}$ and $Q_{N}$. It can be clearly seen that the proposed control yields confinement of vibrations in both cases. However, we notice that the use of $Q_{N}$ leads to faster suppression of vibrations of the sensitive elements and to smoother time response at all nodes. Another important design objective is to reduce the input energy. In order to gain insight into the control energy needed for the simultaneous confinement and suppression, we suggest the use of the following input norm

$$
\|u(t)\|_{2}=\|\bar{M} \ddot{X}+\bar{C} \dot{X}+\bar{K} X\|_{2}=\sqrt{\sum_{i=1}^{n_{u}} u_{i}(t)^{2}}
$$



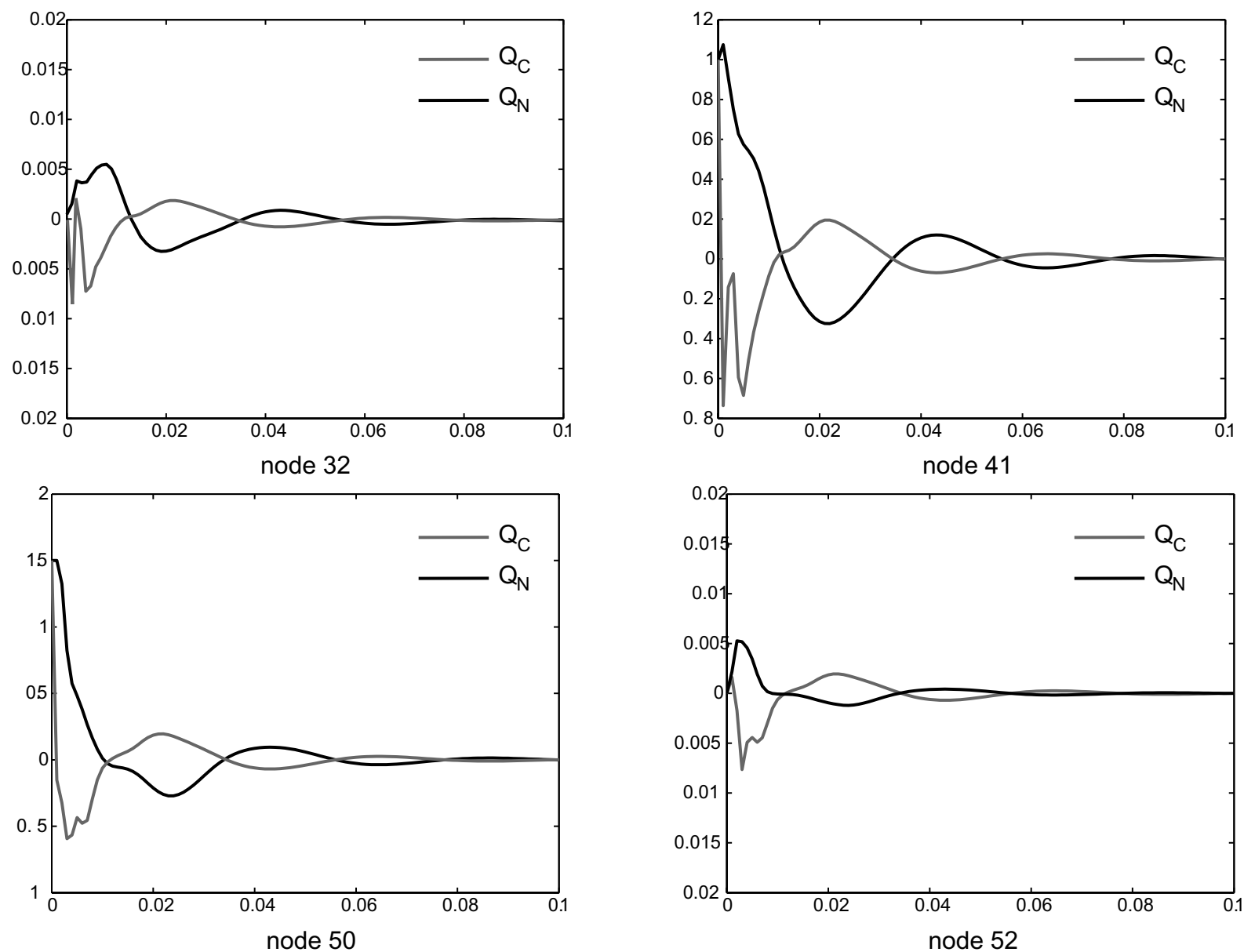

Fig. 2. Plate responses at selected nodes using both forms of $Q$.

Figure 3-a displays the time histories of the input norms associated with the two forms of the pseudo-modal matrix. We observe that the feedback input needed using $Q_{N}$ requires much smaller amplitudes. Similar observations can be made for different damping ratios (see Fig. 3-b and 3-c, which correspond to $\zeta_{i}=0.1$ and $\zeta_{i}=0.5$, respectively).

In order to investigate the effect of altering the natural frequencies associated with the controlled structure, we set these frequencies to 10 and 20 times less than those of the uncontrolled structure. The resulting responses for both forms of $Q$ are displayed in Fig. 4. As expected, reducing the plate natural frequencies leads to slower dynamics, and thus, the proposed control strategy allows the specification of the level of confinement and its rate.

As stated previously, the $\beta_{i j}$ coefficients, with values from 0 to 1 , play a key role in confining the vibrations, and thus, redistributing the energy within the structure. Lowering the value of $\beta_{i j}$ results in the reduction of the amplitude of vibration at the $i^{\text {th }}$ node associated with the $j^{t h}$ mode. This can be easily seen in Fig. 5 where the amplitude of vibration at node 32 is shown for $\beta_{32, j}=0.1, \beta_{32, j}=0.05$ and $\beta_{32, j}=0.01$.

In what follows, the damping coefficients $\zeta_{i}$ are all set to 0.3 , while the natural frequencies of the structure are kept unaltered.

\subsection{Reduced Set of Actuators (RSA)}

For the purpose of comparing the plate response to using a full set of actuators with that using a reduced set of actuators, we consider the plate shown in Fig. 6. The structure is assumed to be initially disturbed such that $X_{40}(0)=1$ and the remaining nodal initial displacements and velocities are zeros. It is also assumed that the plate 

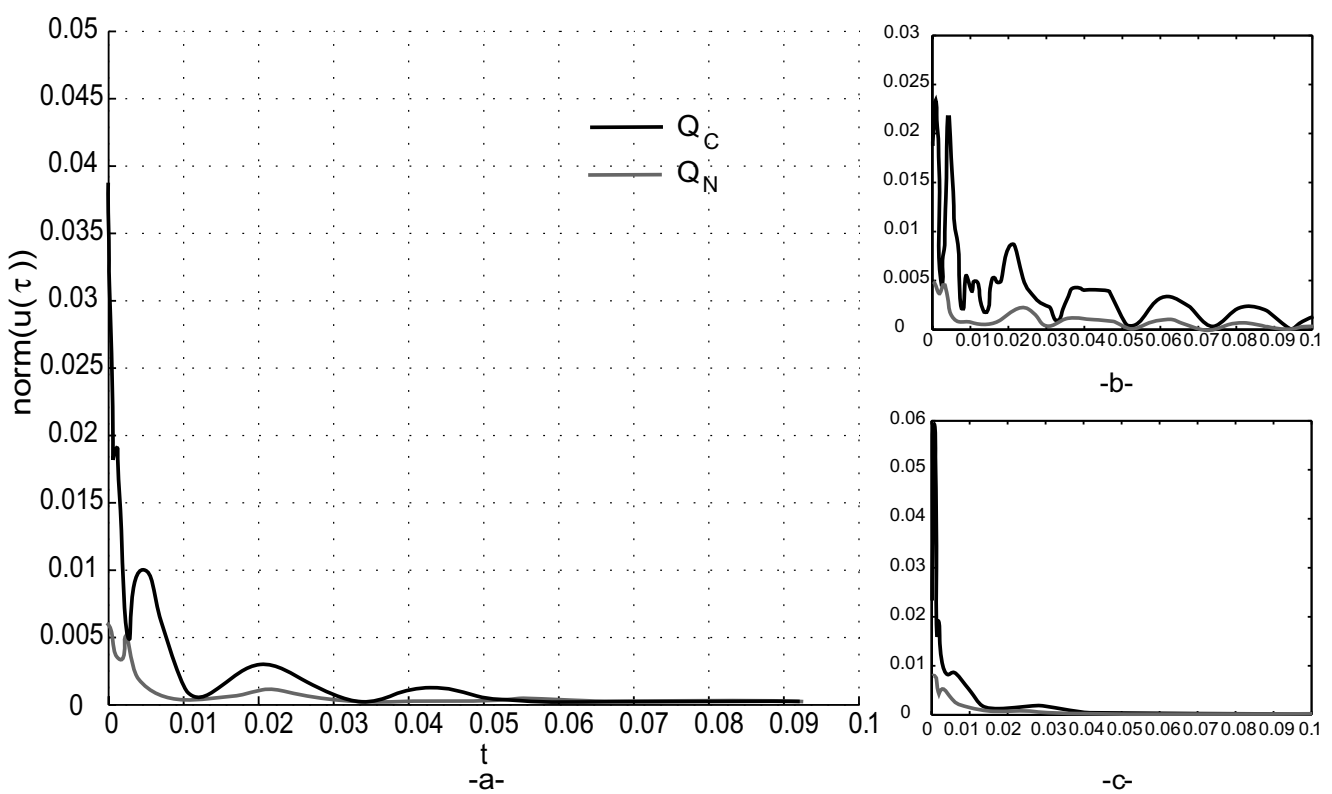

Fig. 3. Norm of the feedback input for different acts of damping ratios.
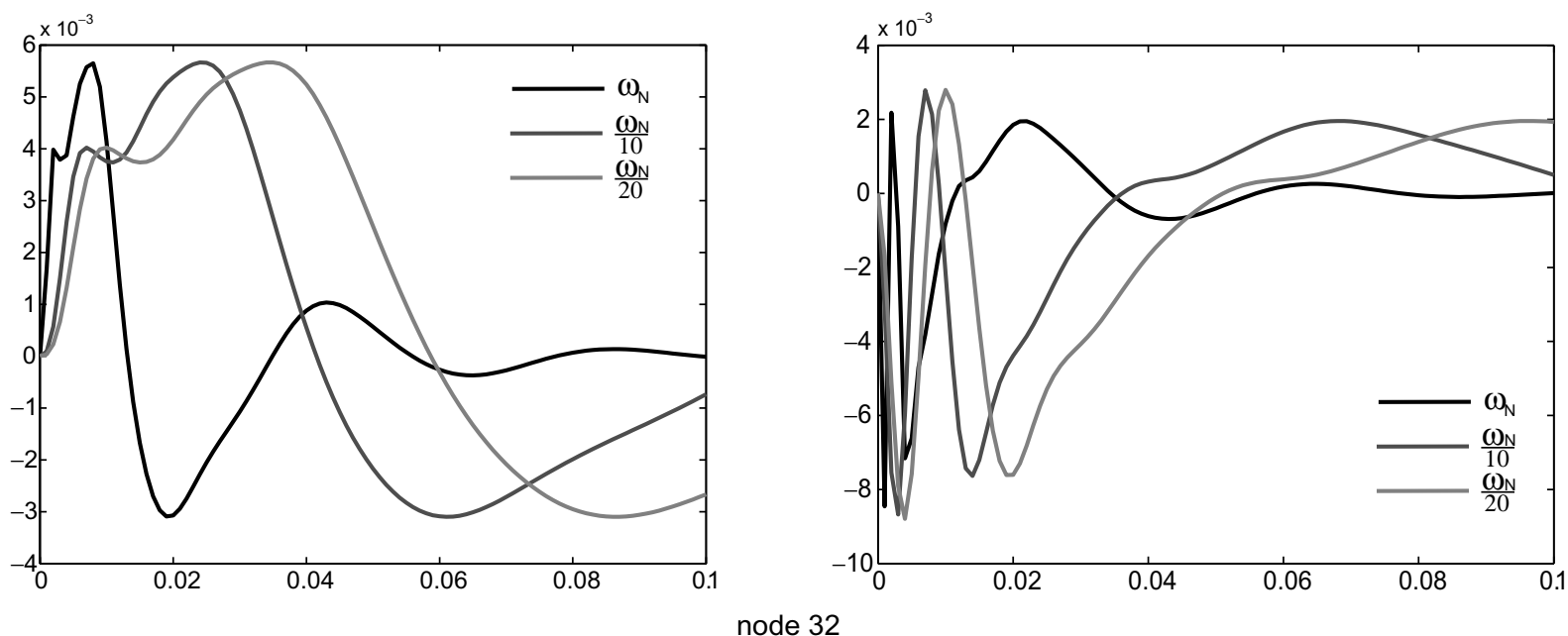

Fig. 4. Effect of altering the natural frequencies at selected nodes.

consists of two subsystems: $S_{1}=\{30,31,32,39,40,41,48,49,50\}$ grouping the sensitive nodes and $S_{2}$ containing the remaining parts of the structure. The subdivision of the structure into two subsystems allows the reduction of the number of actuators for cost-effectiveness purposes.

In order to compare both cases, we examine the resulting displacements of the initially-perturbed node 40 and selected sensitive nodes.

Figure 7 shows clearly that the confinement performance in both cases is satisfactory. The use of a reduced number of actuators yields the desired simultaneous confinement and suppression of vibrations, while the use of a full set of actuators results in slightly better suppression. The feedback inputs needed to achieve the confinement and suppression of vibrations in each case are displayed in Fig. 8. Although they are different, we note that both feedback inputs are comparable. It is clear that using a reduced set of actuators (10 actuators instead of 25) achieves acceptable suppression of vibrations. 


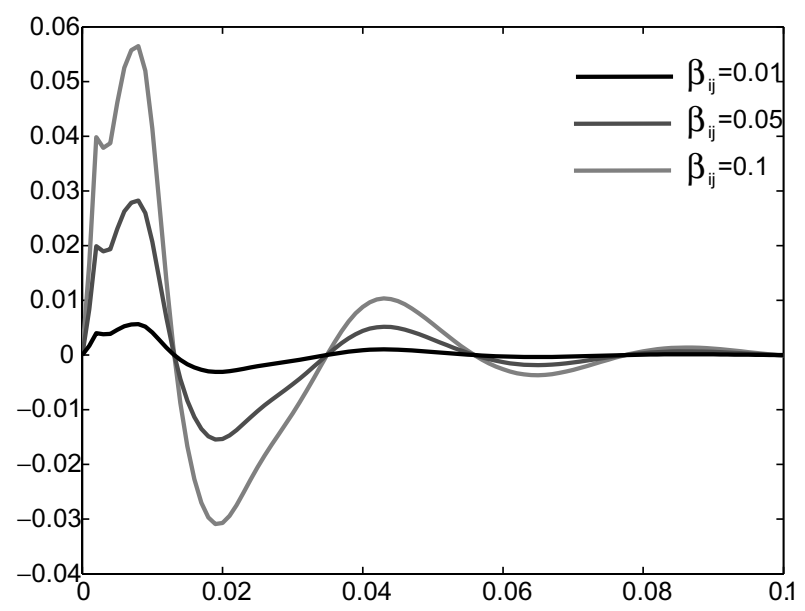

Fig. 5. The effect of the $\beta_{i j}$ coefficients.

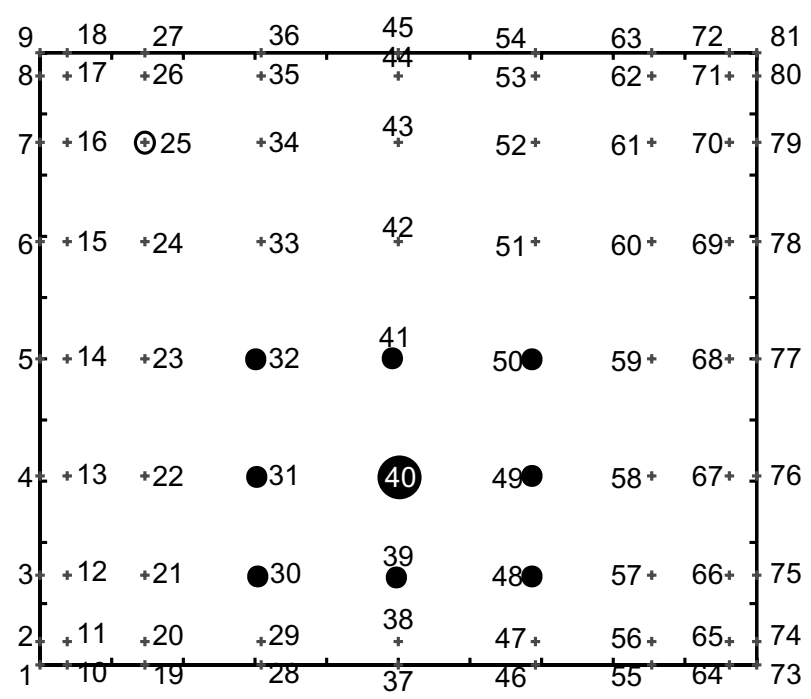

Fig. 6. Actuator locations in the two subsystems.

\subsection{Control of the vibrational energy flow}

Here, we show that it is possible to specify paths for vibration confinement by setting different values for $\beta_{i j}$ of the pseudo-modal matrix $Q_{N}$. As mentioned previously, the values of the $\beta_{i j}$ coefficients are set from 0 to 1 , depending on how sensitive the nodes are. A value close to 0 corresponds to a very sensitive node, whereas 1 corresponds to a nonsensitive node. In order to demonstrate this, we consider a plate subjected to an impact such that $\dot{X}_{39}(0)=0.005$ and the remaining nodal initial displacements and velocities are zeros. We assume two different confinement paths:

- Case 1: the vibrational energy flows to the right side of the plate along a straight line (Fig. 9-a)

- Case 2: the vibrational energy flows through one of the plate's diagonals (Fig. 9-b)

The corresponding sets of the $\beta_{i j}$ coefficients are as shown in Fig. 9-a and 9-b. We note that the excited node belongs to the desired energy path in the first case whereas it does not in the second case. The 3D representations of the corresponding time responses of the plate are displayed in Figs 10 and 11. These simulations show that the desired simultaneous confinement and control of the vibrational energy flow are achieved in both cases. 

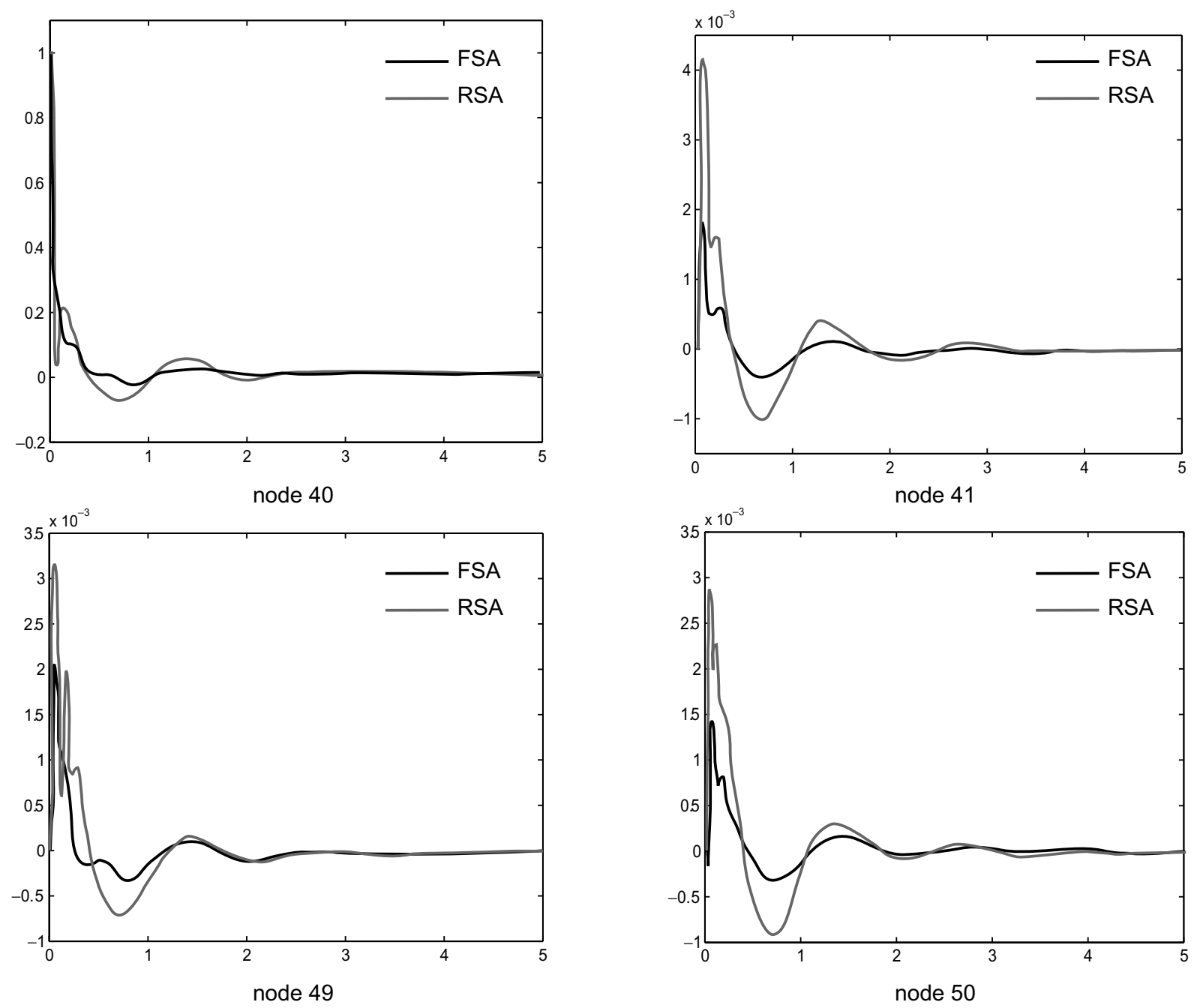

Fig. 7. Plate responses at selected nodes using the FSA and the RSA strategies.

The present simulations show clearly the feasibility of controlling the flow path of the confined energy. Nevertheless, it is of interest to identify the set of paths that best confine the vibratory motion. This can be defined as follows: for a given set of sensitive parts, what is the set of paths that best confine the vibrational energy with the lowest cost possible of supplied input energy?

To illustrate such concept, we consider the confinement configurations represented in Fig. 12. We then compare the input norm needed for these different confinement configurations. In fact, as illustrated by Fig. 13, the second case (flow along both diagonals of the plate) leads to a reduction of $40 \%$ of the input feedback required in the first case. It can be concluded that larger total lengths of the energy path(s) yield smaller magnitudes of the control efforts and faster energy decays.

\section{Conclusions}

In this paper, the vibration confinement and energy flow control of $2 \mathrm{D}$ flexible structures were studied. We proposed an HQM discretized-model-based feedback strategy, aiming at confining and suppressing simultaneously the vibrations. A new pseudo-modal matrix, derived from the computed eigenvectors of the HQM-discretized model, 


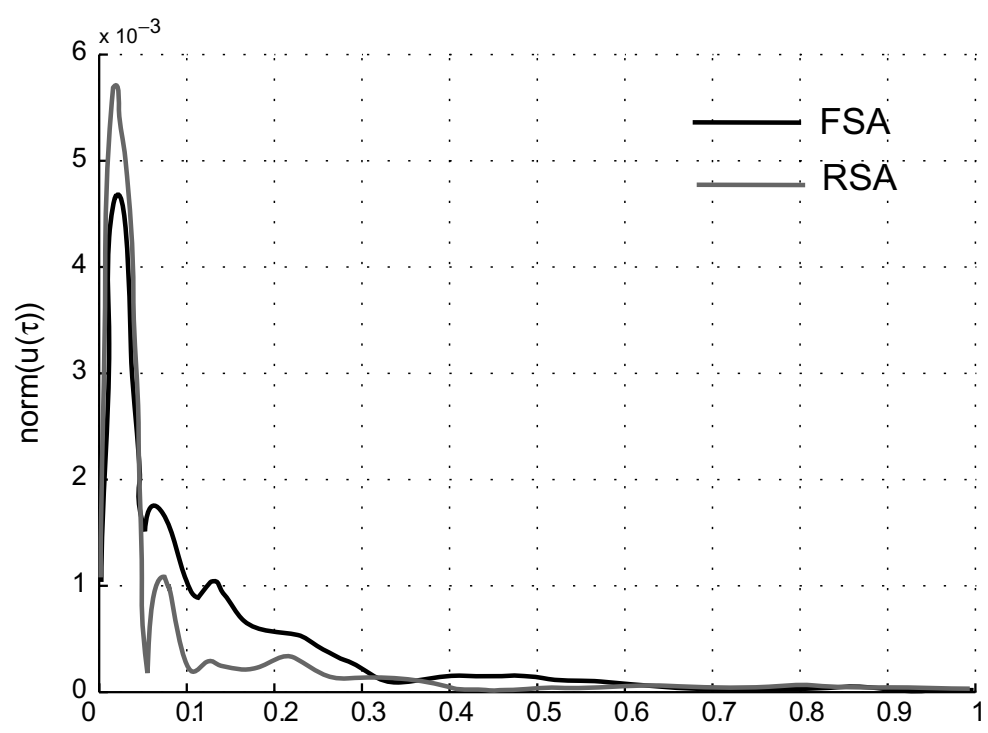

Fig. 8. Norm of the feedback input using full and reduced sets of actuators.

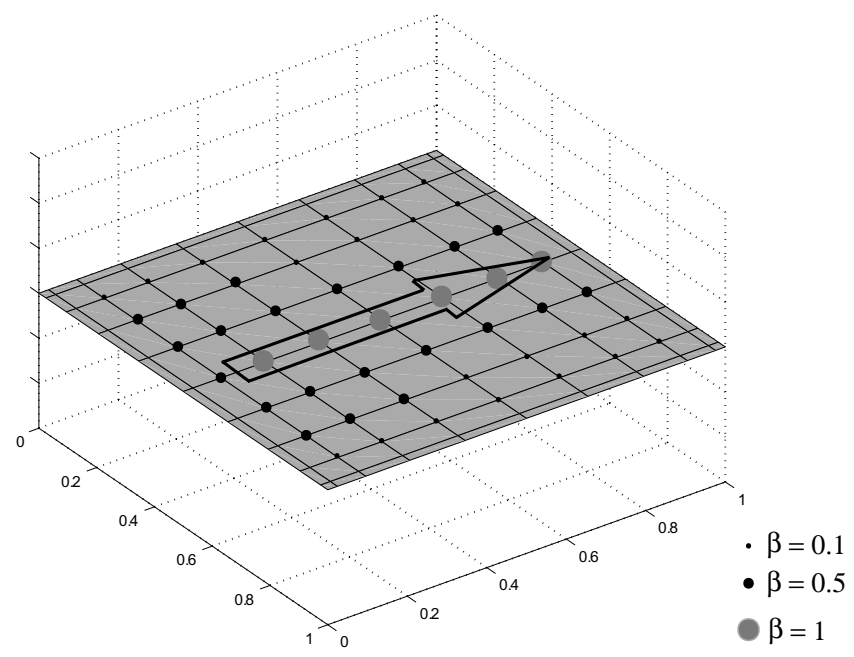

Path 1

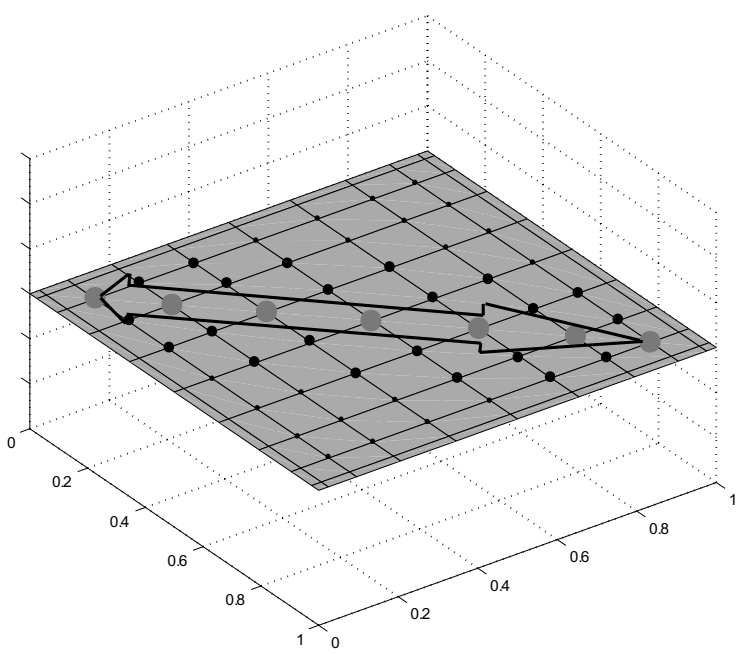

Path 2

Fig. 9. Confinement configuration and corresponding energy flow path.

was introduced to enhance the confinement of vibrations and its suppression. Simulations were presented to show the efficacy of the proposed control law and the effects of its parameters on the simultaneous confinement and suppression of vibrations. In addition, we conducted a set of simulations to investigate the flow control of vibrational energy during the confinement-suppression process. We found that the energy flow can be regulated via a set of control parameters for different confinement configurations. We concluded that longer energy paths yield smaller magnitude of the control efforts and faster energy decay. Confining the vibratory motion and regulating its flow within the structure for the purpose of energy harvesting constitute the focal interest of the authors' future research. For this, there is a need for developing mathematical expressions for the energy flow of vibration for such regulation. 

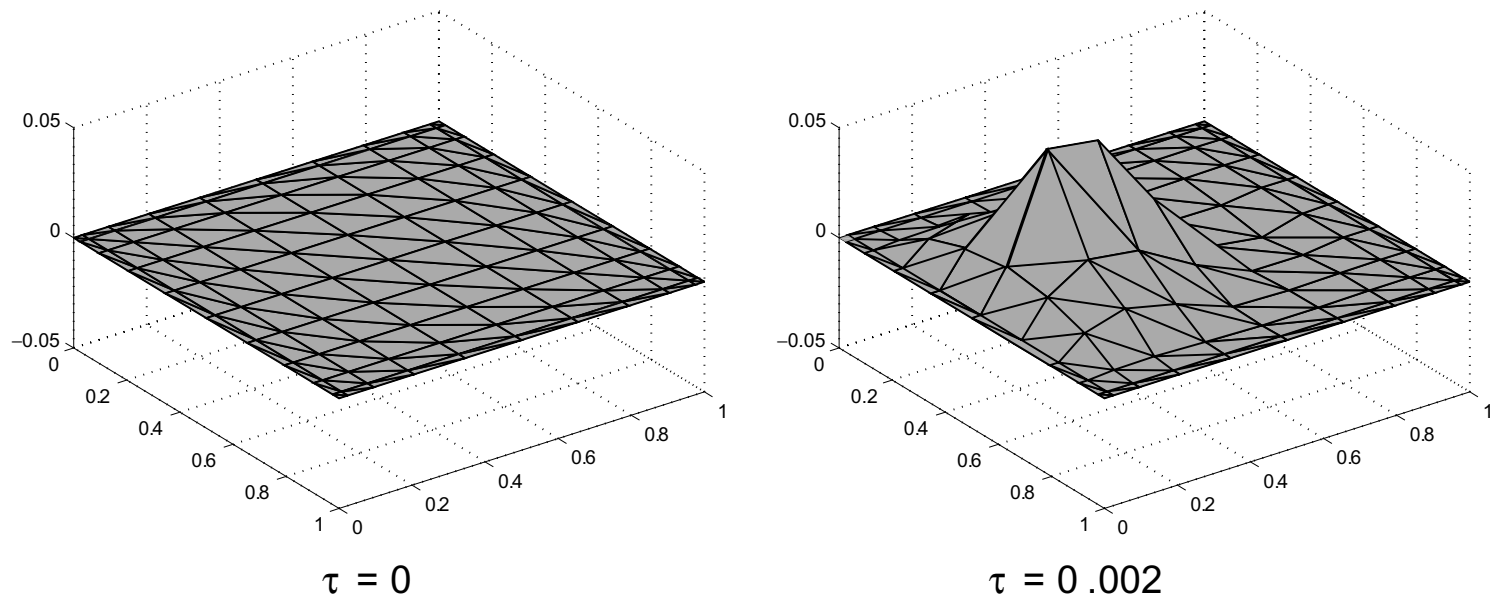

$$
\tau=0.002
$$
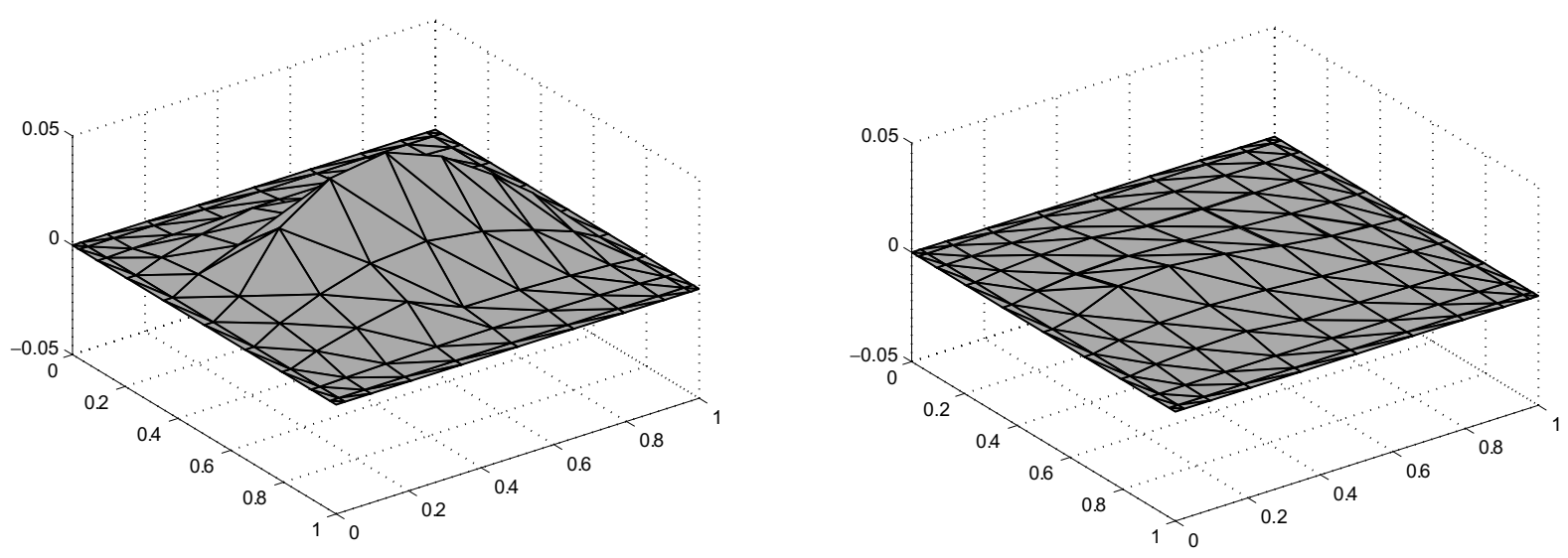

$\tau=0.007$

$\tau=0.018$
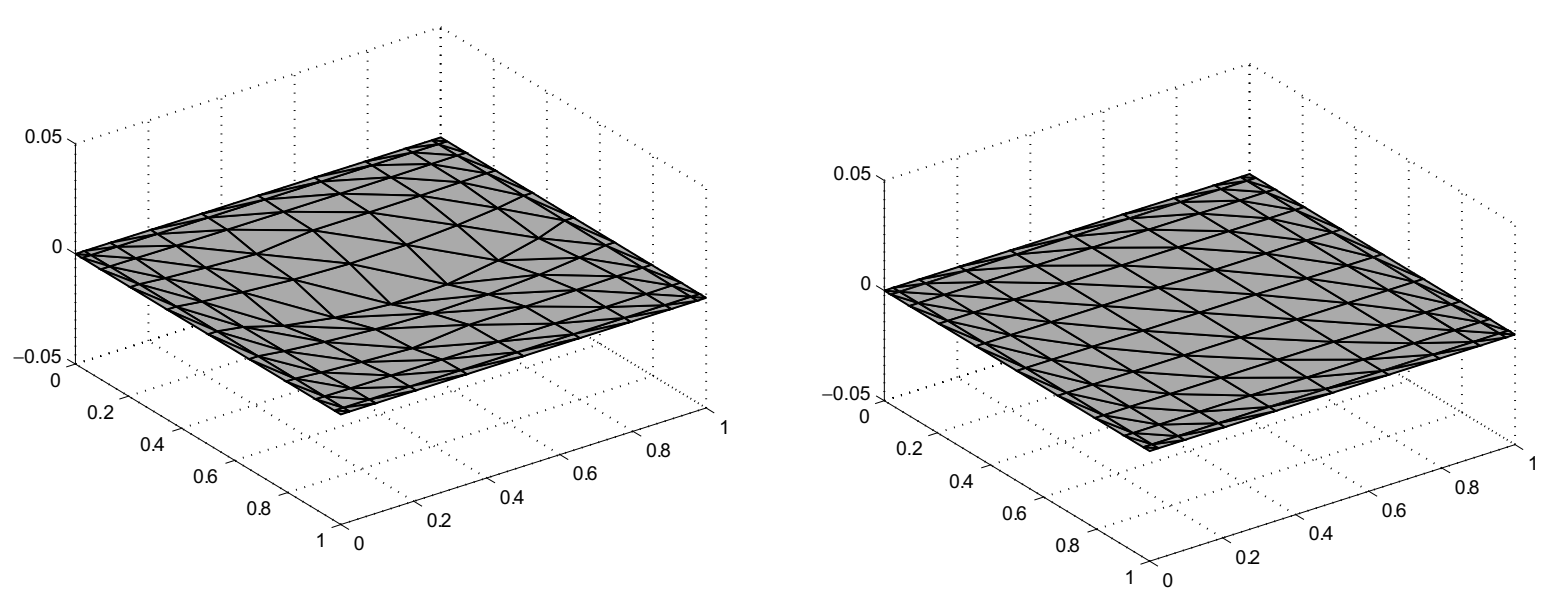

$\tau=0.02$

$$
\tau=0.1
$$

Fig. 10. Plate time response at selected times (confinement path 1). 

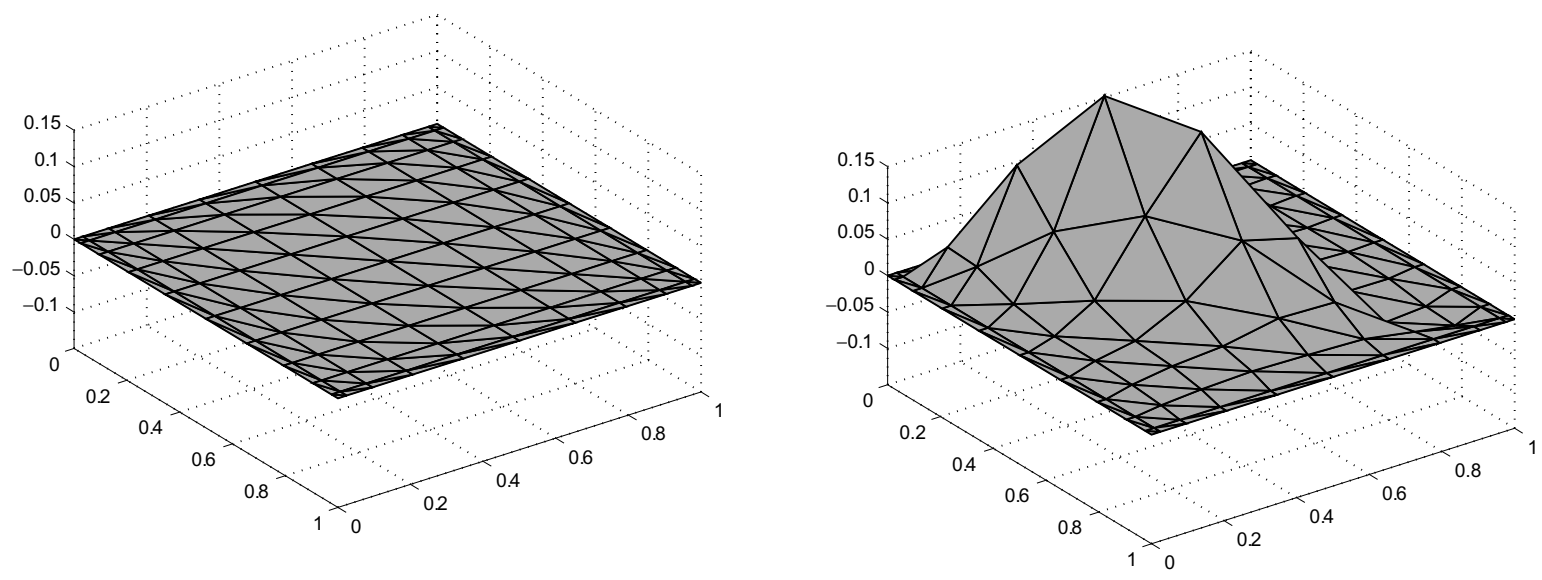

$$
\tau=0
$$

$$
\tau=0.004
$$
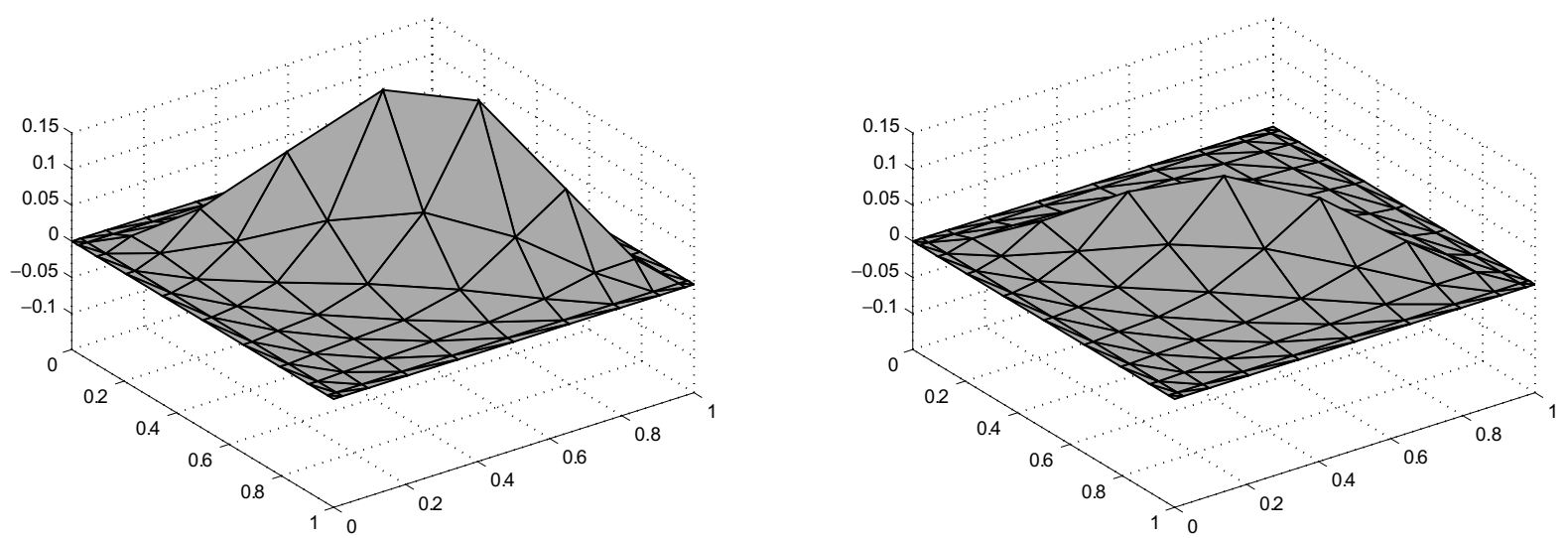

$$
\tau=0.01
$$

$$
\tau=0.015
$$

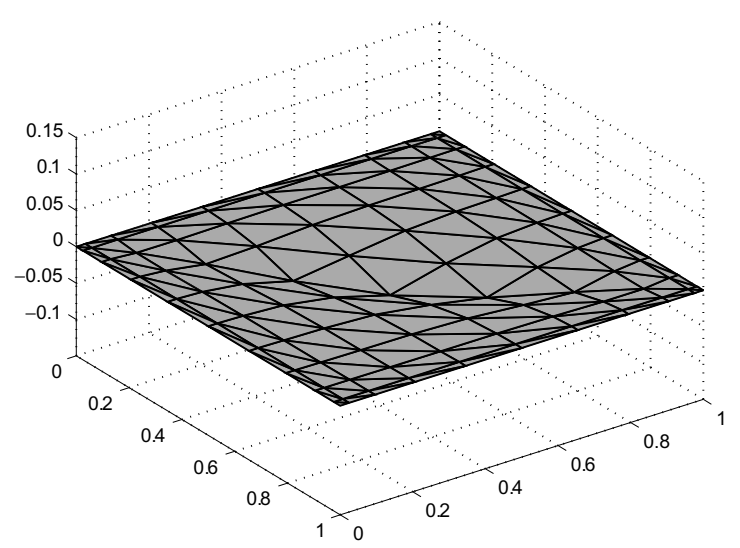

$\tau=0.02$

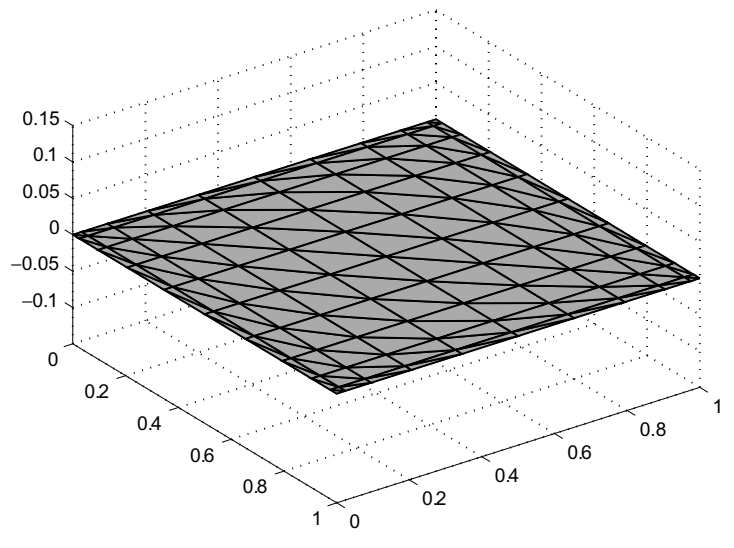

$\tau=0.1$

Fig. 11. Plate time response at selected times (confinement path 2). 


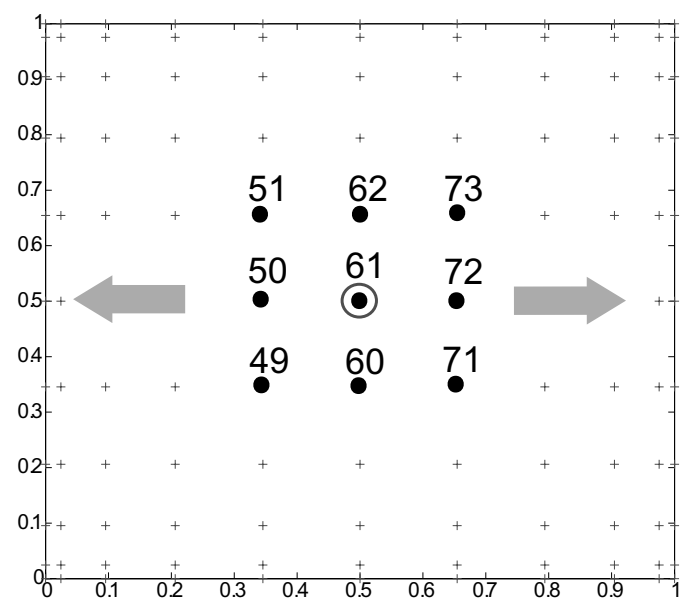

Path 1

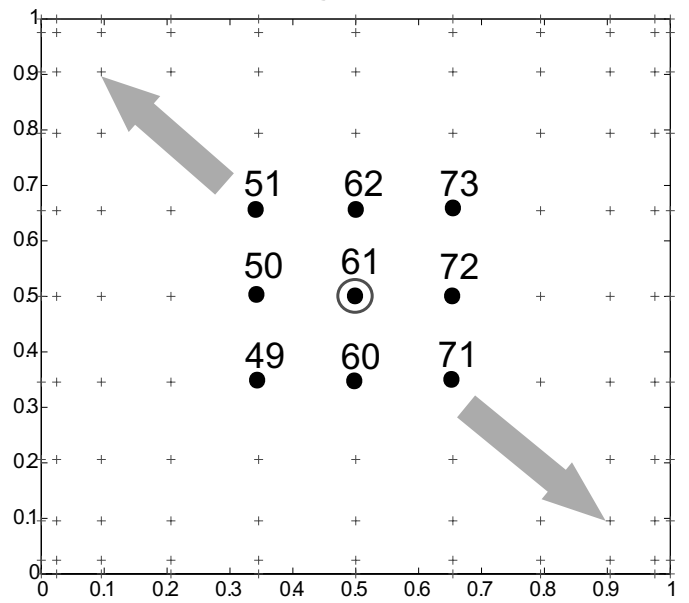

Path 3

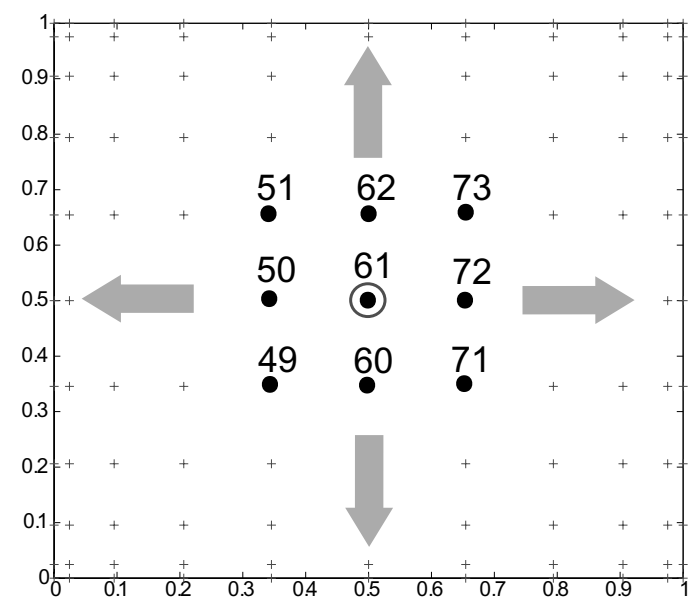

Path 2

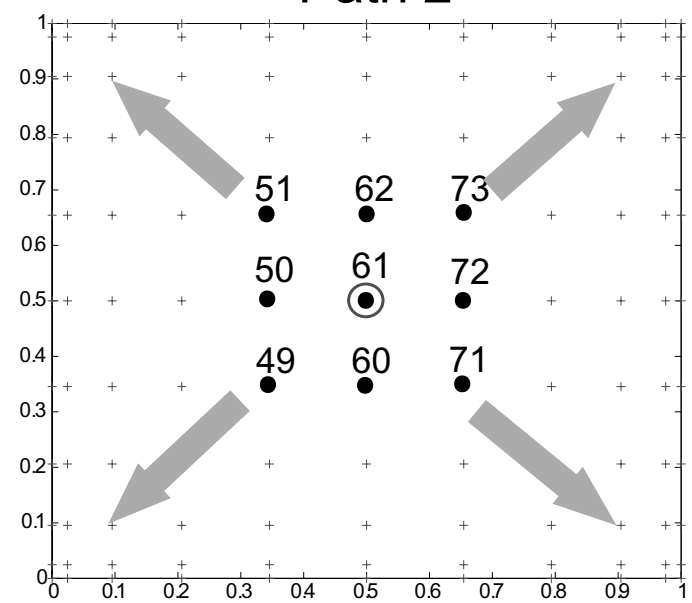

Path 4

\section{$\bigcirc$ excitation node $\bullet$ sensitive node}

Fig. 12. Different paths for energy flow.

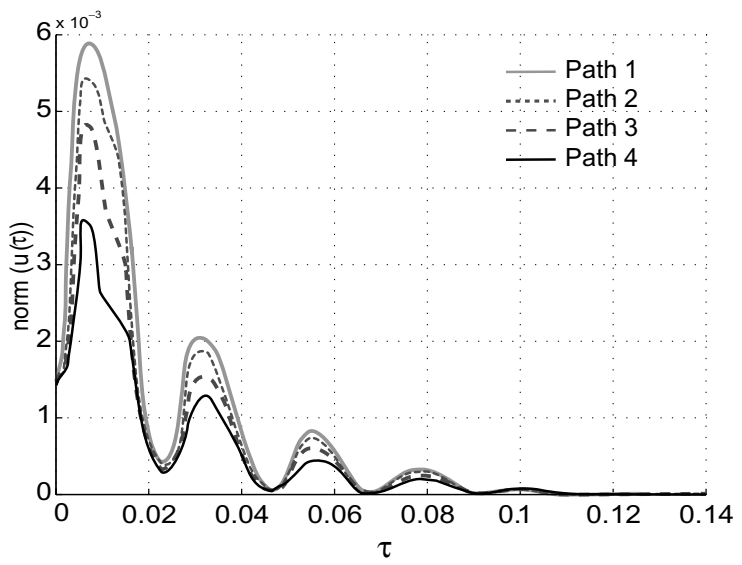

Fig. 13. Normalized input feedback associated with the different energy flow paths. 


\section{References}

[1] C.M. Harris and A.G. Piersol, eds, Harris Shock and Vibration Handbook, 5 edition, McGraw-Hill, 2002.

[2] C.H. Hodges, Confinement of vibration by structural irregularity, Journal of Sound and Vibration 82 (1982), $411-424$.

[3] A.F. Vakakis, Passive spatial confinement of impulsive responses in coupled non linear beams, AIAA Journal 32 (Year), $1902-1910$.

[4] D. Allaei, Performance comparison between vibration control by confinement and conventional control techniques. Proceedings of the ASME 16th Biennial Conference on Mechanical Vibration and Noise, Sacramento, CA, September 1997, 14-17.

[5] M.M. Fahmy and J. O'Reilly, On eigenstructure assignment in linear multivariable system, IEEE Transactions on Automatic Control 27 (1982), 690-693.

[6] J. Kautsy, N.K. Nichols and O. Van Dooren, Robust pole assignment in linear state feedback, International Journal of Control 41 (1985), 1229-1245.

[7] J.N. Juang, K.B. Lim and J.L. Junkins, Robust eigensystem assignment for flexible structures, Journal of Guidance, Control, and Dynamics 12 (1989), 311-317.

[8] P.G. Maghami and S. Gupta, On the eigensystem assignment with dissipativity constraints. Proceedings of the American Control Conference, San Francisco, CA, June 1993, 1271-1275.

[9] P.G. Maghami, J. Juang and K.B. Lim, Eigensystem assignment with output feedback, Journal of Guidance, Control, and Dynamics 15 (1993), 531-536.

[10] F.J. Shelley and W.W. Clark, Experiments in eigenstructure assignment for active mode localization in a flexible beam. Proceedings of the American Control Conference, Albuquerque, New Mexico, June 1997.

[11] B.K. Song and S. Jayasuriya, Active vibration control using eigenvector assignment for mode localization. Proceedings of the American Control Conference, San Francisco, CA, June 1993, 1020-1024.

[12] J. Shaw and S. Jayasuriya, Arbitrary assignment of eigenvectors with state feedback, ASME Journal of Dynamic Systems, Measurement, and Control 114 (1992), 721-723.

[13] W.W. Clark and F.J. Shelley, Active mode localization in distributed parameter systems with consideration of limited actuator placement, part I: Theory; part ii: Simulations and experiments, Journal of Vibration and Acoustics 122 (1995), 160-168.

[14] A. Preumont, Vibration Control of Active Structures: An Introduction, Kluwer Academic Publishers, 2004.

[15] Y. Meyer and M. Collet, Mixed control for robust vibration isolation: Numerical energy comparison for an active micro suspension device, Smart Materials and Structures 16 (2007), 1361-1369.

[16] Y.R. Hu and A. Ng, Active robust vibration control of flexible structures, Journal of Sound and Vibration 288 (2005), 43-56.

[17] S. Choura and A.S. Yigit, Vibration confinement in flexible structures by distributed feedback, Journal of Computers and Structures $\mathbf{5 4}$ (1995), 531-540.

[18] S. Choura and A.S. Yigit, Confinement and suppression of structural vibrations, ASME Journal of Vibration and Acoustics 123 (2001), 496-501.

[19] E. Civalek, Application of differential quadrature (dq) and harmonic differential quadrature (hdq) for buckling analysis of thin isotropic plates and elastic columns, Engineering Structures 26 (2004), 171-186.

[20] G. Karami and P. Malekzadeh, An efficient differential quadrature methodology for free vibration analysis of arbitrary straight-sided quadrilateral thin plates, Journal of Sound and Vibration 263 (2003), 415-442.

[21] G. Karami, S.A. Shahpari and P. Malekzadeh, DQM analysis of skewed and trapezoidal laminated plates, Composite Structures 59 (2003), 393-402.

[22] M. Ulker and O. Civalek, Application of harmonic differential quadrature (hdq) to deflection and bending analysis of beams and plates, $F$. U. Fen ve Muhendislik Bilimleri Dergisi (2004), 221-231.

[23] R.A. Horn and C.R. Johnson, Topics in Matrix Analysis, Cambridge University Press, 1994.

[24] S. Choura and A.S. Yigit, Active control of linear time-varying structures by confinement of vibrations, Journal of Vibration and Control 11 (2005), 89-102.

[25] S. Choura, Control of flexible structures with the confinement of vibrations, ASME Journal of Dynamic Systems, Measurement, and Control 117 (1995), 155-164. 

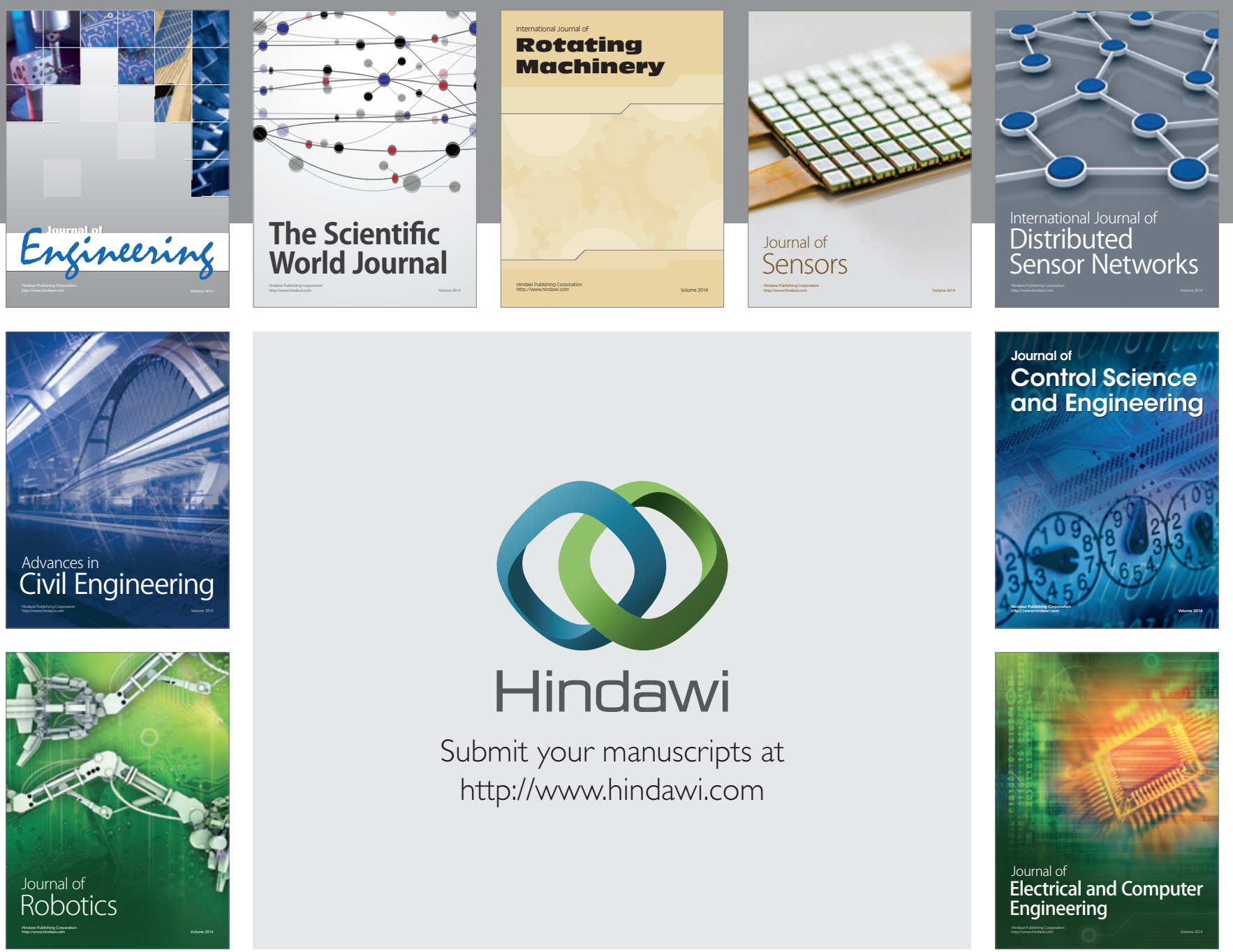

Submit your manuscripts at

http://www.hindawi.com
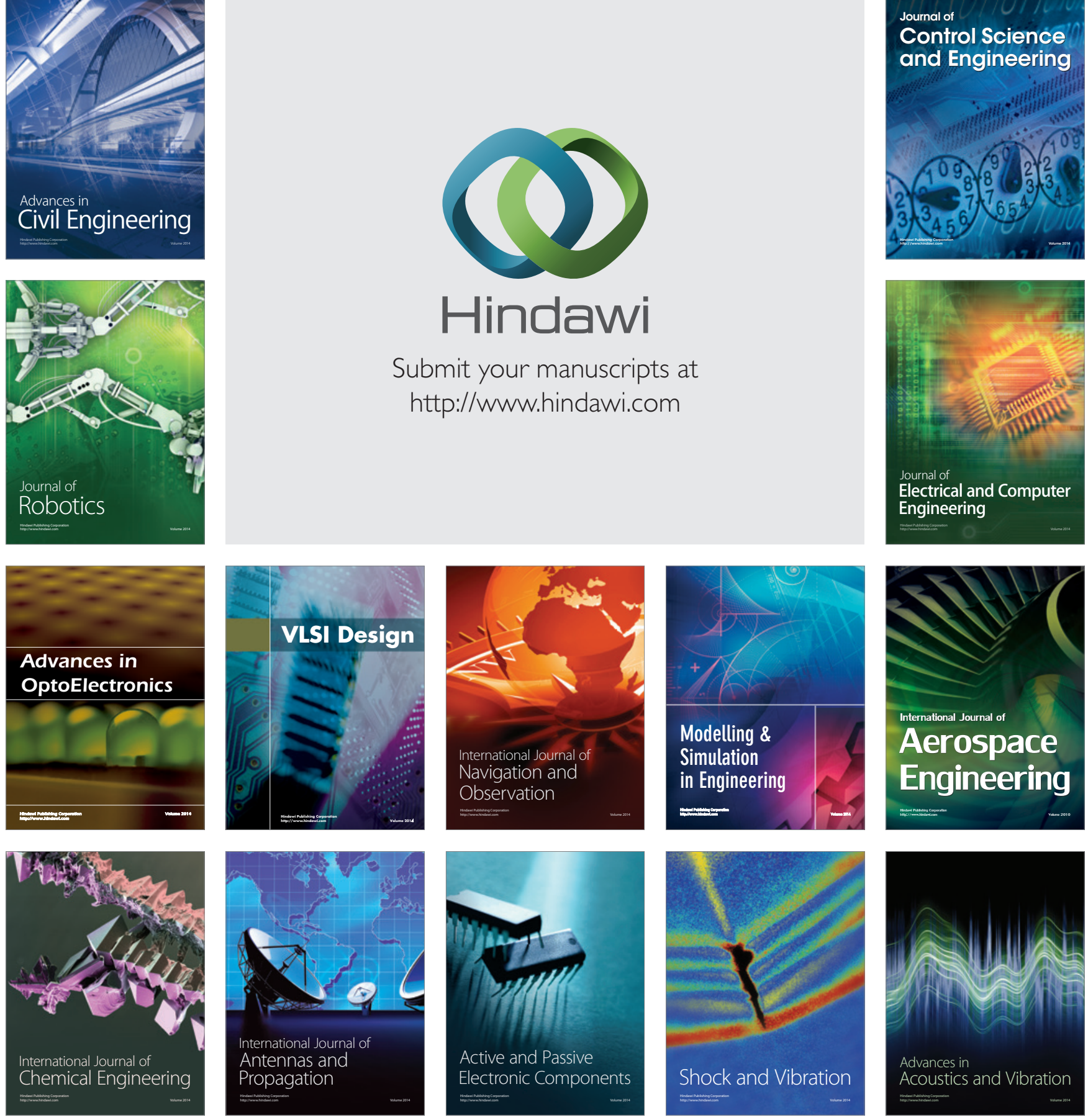